\title{
LAS BUENAS PRÁCTICAS COMO RECURSO PARA LA ACCIÓN COMUNITARIA: CRITERIOS DE IDENTIFICACIÓN Y BÚSQUEDA ${ }^{1}$
}

\author{
Rita Gradaille Pernas \\ María Belén Caballo Villar \\ Universidad de Santiago de Compostela
}

\begin{abstract}
RESUMEN: En los últimos tiempos, en las investigaciones científicas y en el trabajo cotidiano de diferentes entidades y colectivos, se impone un enfoque basado en la necesidad de visibilizar las buenas prácticas con el fin de identificar aquellos elementos que permiten significarlas como tales, tanto por su excelencia como por su capacidad de hacerlas transferibles.

Existen diferentes criterios que permiten caracterizar una buena práctica, aunque no siempre responden a determinados principios de selección; con todo, son iniciativas dotadas de originalidad que inspiran el concepto de una acción transferible, sustentable e innovadora, homologable a determinados territorios.

En este artículo se argumentan las características que debe cumplir una experiencia para ser considerada como una Buena Práctica, complementando la propuesta con indicadores específicos relativos a la acción comunitaria. En ambos casos se construyen a partir de los planteamientos realizados por organismos internacionales, por entidades que representan el trabajo de las administraciones y por el tercer sector. Además, se hace un recorrido por algunos Bancos de Buenas Prácticas para ponerlos en valor como recurso que ayuda a mejorar las actuaciones en clave pedagógica y social, incrementando la calidad de vida de las personas y las comunidades.
\end{abstract}

PALABRAS CLAVE: Buenas prácticas, acción comunitaria, pedagogía social, educación social, bancos de buenas prácticas.

1. El artículo que presentamos se vincula con los siguientes proyectos de investigación:

"De los tiempos educativos a los tiempos sociales: la construcción cotidiana de la condición juvenil en una sociedad de redes. Problemáticas específicas y alternativas pedagógico-sociales" (Proyecto coordinado EDU2012-39080-C07-00 a 07), cofinanciado por el Fondo Europeo de Desarrollo Regional (FEDER, 2007-2013) en el marco del Plan Nacional de I+D+i con cargo a una ayuda del Ministerio de Economía y Competitividad.

"La conciliación en los tiempos cotidianos de la infancia en Galicia: problemáticas específicas y alternativas pedagógico-sociales y de ocio en las escuelas, familias y comunidades", proyecto financiado por la Xunta de Galicia en la convocatoria de investigadores emergentes 2014 (EM2014/006). 


\title{
GOOD PRACTICES AS A RESOURCE FOR COMMUNITY ACTION: IDENTIFICATION AND SEARCH CRITERIA
}

\begin{abstract}
In the last times, in scientific research and in the daily work of different organizations and groups, based on the need to make visible the good practices in order to identify those elements that allow to mean them like such, both for its excellence and its ability to make them transferable.

There are different criteria that allow characterizing a good practice, although not always respond to certain principles of selection; they are endowed originality experiences that inspire the concept of a transferable, sustainable and innovative practice that is comparable to certain territories. Hence, some of these initiatives have the aim of providing an overview of types of initiatives that demonstrate new ways to increase creativity, reinventing certain educational activities in order to achieve a more educational, more civic and citizen culture.

This article describes the features that must meet an experience to be considered as a good practice, complementing the proposal with specific indicators for Community action argue. In both cases they are constructed from the proposals made by international bodies, by entities that represent the work of the government and the third sector. While touring Banks of Good Practices managed by institutions, public and private entities is done, etc. with the aim of demonstrating contributions that improve the performances in educational and social key, and increase the quality of life of people and communities.
\end{abstract}

KEYWORDS: Best practices, community action, social pedagogy, social education, best practices banks.

Recibido: 07/05/2015

Aceptado: 22/07/2015

Correspondencia: Rita Gradaille Pernas, Universidad de Santiago de Compostela, Facultad de Ciencias de la Educación, Departamento de Teoría de la Educación, Historia de la Educación y Pedagogía Social, Campus Sur, 15782 Santiago de Compostela. Email: rita.gradaille@usc.es.

\section{INTRODUCCIÓN}

La creciente complejidad de la sociedad contemporánea -que se manifiesta en sus modos de vida plurales, en sus necesidades superpuestas o en la diversidad de opciones- conlleva la proliferación de actuaciones de las que no siempre resulta fácil discernir su pertinencia, eficacia y calidad. Es por ello que, de forma progresiva, tanto en las investigaciones científicas como en la cotidianidad de las administraciones, de las empresas y de las entidades sociales, se impone un enfoque basado en la identificación de buenas prácticas que permitan servir de orientación a la hora de tomar decisiones acertadas para mejorar la realidad social. 
Hacer visibles aquellas acciones que han sido reconocidas tanto por su excelencia como por su capacidad de hacerlas transferibles a otros contextos, contribuye a incrementar la calidad del trabajo y su repercusión; a compartir los aprendizajes, evitando la repetición de errores ya cometidos por otros; a motivar para buscar nuevas y mejores alternativas a problemas y necesidades comunes; a mejorar la imagen interna y externa de la entidad que las impulsa; también a tender puentes entre la investigación, las políticas y la acción social, orientando el desarrollo de nuevas propuestas. Se trata, pues, de contribuir a estimular la reflexión que ayuda a madurar las teorías y las prácticas, generando conocimiento socialmente útil (OIT, 2003; FEAPS, 2007).

Es por ello que tanto los investigadores e investigadoras como los profesionales y el voluntariado que desarrollan su labor en administraciones públicas, empresas, fundaciones, organizaciones no gubernamentales, asociaciones, etc., encuentran en las buenas prácticas un referente de interés para orientar y mejorar su trabajo.

Al margen de las debilidades conceptuales identificadas en el terreno de las buenas prácticas, existe cada vez un mayor consenso respecto a su consideración como contribuciones valiosas, caracterizadas por tener un impacto tangible en la mejora de la calidad de vida de las personas y de las comunidades; por estar social, cultural, económica y ambientalmente orientadas a la sostenibilidad. Se trata, pues, de acciones -también de metodologías o herramientas- en las que hay reflexión, intencionalidad, cierta sistematicidad en su diseño, desarrollo y evaluación, así como flexibilidad para adaptarse a la realidad en función de los cambios que se van produciendo. Es por ello que una buena práctica tiene capacidad para satisfacer necesidades y expectativas mejorando los estándares de un servicio, siempre en consonancia con criterios éticos, técnicos y conforme a la misión y valores de la entidad que la promueve (FEAPS, 2007).

\section{Criterios para identificar una Buena Práctica}

Diferentes organismos y entidades de carácter nacional e internacional han realizado importantes esfuerzos para acotar los criterios que permiten adjetivar una práctica como "buena", aunque éstos siempre han de ser entendidos como directrices generales que pueden variar o matizarse en función del ámbito al que se circunscriba la acción. También se debe tener en cuenta que una buena práctica no alude a acciones perfectas, sin fallos, sino a iniciativas que cumplen una serie de requisitos que las convierten en experiencias valiosas por su capacidad de orientar el presente y el futuro.

Tomaremos como punto de partida la propuesta de la UNESCO, que en el marco del programa Management of Social Transformations (2003) concreta cuatro rasgos básicos para identificar lo que denomina como "best practicies" en el ámbito social: la innovación, la eficacia, la sostenibilidad y la replicabilidad de las iniciativas. Estos cuatro elementos de partida han sido incorporados en la mayor parte de las sistematizaciones coetáneas o posteriores. Es por ello que, desde una perspectiva metodológica, hemos optado por recoger en la tabla 1 esta propuesta junto con la de la Organización Internacional del Trabajo (2003) -ambos organismos de carácter internacional-; la que realiza el Observatorio Internacional de la Democracia Parti- 
cipativa (2015), y, en el ámbito educativo, la Agencia Andaluza de Evaluación Educativa (2012), pues representan la perspectiva de las administraciones públicas (municipales en el primer caso y autonómica en el segundo); también la que avanza la Fundación CEPAIM (2014), una ONG que trabaja en clave comunitaria e intercultural por la convivencia y la cohesión social y que ejemplifica la labor del tercer sector. Quedan así visibilizados diferentes agentes de la acción comunitaria para comenzar el análisis del sentido y significado de los indicadores substantivos más reiterados.

Tabla 1. Criterios para identificar una buena práctica

\begin{tabular}{|c|c|c|c|c|}
\hline $\begin{array}{l}\text { UNESCO } \\
(2003)\end{array}$ & OIT (2003) & $\begin{array}{c}\text { Agencia } \\
\text { andaluza de } \\
\text { evaluación } \\
\text { educativa } \\
\text { (2012) }\end{array}$ & $\begin{array}{c}\text { CEPAIM } \\
(2014)\end{array}$ & $\begin{array}{l}\text { Observatorio } \\
\text { Internacional de } \\
\text { la Democracia } \\
\text { Participativa } \\
(2015)\end{array}$ \\
\hline $\begin{array}{l}\text { Innovación } \\
\text { Efectividad } \\
\text { Sostenibilidad } \\
\text { Replicabilidad }\end{array}$ & $\begin{array}{l}\text { Innovación- } \\
\text { creatividad } \\
\text { Eficacia- } \\
\text { impacto } \\
\text { Replicabili- } \\
\text { dad } \\
\text { Sostenibilidad } \\
\text { Pertinencia } \\
\text { Ética y res- } \\
\text { ponsable } \\
\text { Trabajo en } \\
\text { red } \\
\text { Eficiente }\end{array}$ & $\begin{array}{l}\text { Hechos consta- } \\
\text { tables } \\
\text { Responde a las } \\
\text { necesidades } \\
\text { Innovación } \\
\text { Secuenciada y } \\
\text { reflexiva } \\
\text { Documentada } \\
\text { Efectiva y efi- } \\
\text { ciente } \\
\text { Participación } \\
\text { ciudadana } \\
\text { Recursos sufi- } \\
\text { cientes y defi- } \\
\text { nidos } \\
\text { Seguimiento ri- } \\
\text { guroso; retroali- } \\
\text { mentación } \\
\text { Código ético }\end{array}$ & $\begin{array}{l}\text { Sostenible } \\
\text { Efectiva } \\
\text { Innovadora } \\
\text { Transferible } \\
\text { Genera cohe- } \\
\text { sión } \\
\text { Potencia el } \\
\text { empodera- } \\
\text { miento } \\
\text { Redes sociales } \\
\text { Perspectiva de } \\
\text { género }\end{array}$ & $\begin{array}{l}\text { Objetivos relacio- } \\
\text { nados con la parti- } \\
\text { cipación } \\
\text { Innovación } \\
\text { Transferibilidad } \\
\text { Factibilidad } \\
\text { Planificación y } \\
\text { prácticas en el go- } \\
\text { bierno local } \\
\text { Corresponsabilidad } \\
\text { Liderazgo político } \\
\text { Responsabilidades } \\
\text { definidas } \\
\text { Proceso educativo } \\
\text { Impacto y transfor- } \\
\text { mación Evaluación } \\
\text { Devolución de la } \\
\text { información }\end{array}$ \\
\hline
\end{tabular}

Fuente: Elaboración propia.

\section{Innovación}

Se concibe la innovación como una mejora del servicio prestado, aumentando la capacidad global de una estructura organizativa para dar soluciones a determinados problemas; al tiempo que introduce modos creativos de hacer en la medida en que rompen con los hábitos y las referencias del pasado. En definitiva, incorpora elementos o mejora los existentes para perfeccionar el funcionamiento interno o externo de una determinada acción. 
En relación al criterio de innovación, existen diferentes niveles que se pueden considerar en una Buena Práctica:

- Nivel funcional: consiste en desarrollar nuevas tareas, procesos y modelos que surgen de la dinámica del proceso.

- Nivel estructural: emergen sistemas organizativos que optimizan el funcionamiento real y consolidan marcos de actuación alternativos.

- Nivel de comportamientos: modifican los patrones de conducta y una nueva cultura organizativa basada en la negociación y resolución de conflictos.

- Nivel relacional: se transforman las redes de relación y las formas de interacción con el entorno.

- Nivel metodológico: cambian los elementos básicos de un sistema de gestión con la finalidad de mejorar su funcionamiento. Se pueden, pues, modificar los recursos técnicos, financieros y humanos; así como la estructura organizacional y los procesos.

\section{Eficacia/eficiencia}

La eficacia hace referencia a la capacidad para lograr lo que se ha determinado. Por tanto, una iniciativa es eficaz cuando demuestra un impacto positivo y tangible en el territorio, en la comunidad en la que se desarrolla, provocando unos efectos observables y valiosos en el ámbito donde se ha Ilevado a cabo dicha actuación. La incidencia se suele evaluar mediante estudios que, en base a indicadores de carácter cuantitativo y cualitativo, analizan la situación anterior al desarrollo de una iniciativa y la comparan con la posterior; entendiendo que ha habido impacto cuando la situación posterior presenta variaciones significativas -y en positivo- respecto a la previa, generando beneficios individuales y sociales.

Se hace, entonces, imprescindible, un sistema de evaluación que ayude a testar los efectos producidos por las actuaciones desarrolladas en relación a los objetivos previstos, con el fin de constatar las desviaciones y redefinir los objetivos y las medidas propuestas. Es por ello que la flexibilidad, como capacidad de reajuste cuando se producen cambios, y una actitud de actualización permanente, son básicos para que las experiencias sigan funcionando y produciendo resultados de calidad.

Por su parte, se entiende por eficiencia la relación entre los recursos utilizados en un proyecto o acción y los logros conseguidos con el mismo; esto es, alcanzar un objetivo ajustando adecuadamente los medios. En este sentido, una Buena Práctica debería ser eficaz porque satisface los objetivos propuestos; y eficiente, porque lo consigue utilizando del mejor modo los recursos económicos, materiales y humanos disponibles para tal fin.

\section{Sostenibilidad}

Una iniciativa es sostenible cuando cuenta con una estructura organizativa, técnica, económica y social que permiten su funcionamiento de forma armónica a lo largo del tiempo. A este respecto, resulta importante la permanencia o capacidad de 
mantener un servicio con una calidad aceptable durante un período razonable, pues el logro de objetivos a corto, medio y largo plazo permite visibilizar un impacto sostenido, produce efectos duraderos.

Por su parte, la OIT (2003) define la sostenibilidad como la evaluación del potencial de continuidad de la experiencia después de finalizado el período previsto para su implementación o su potencialidad para crear nuevas acciones a partir de la inicial. Evoca, pues, perspectivas de readaptación y estabilidad de los proyectos.

En el campo concreto de la acción comunitaria se entiende que la sostenibilidad de las experiencias guarda una estrecha correspondencia con las acciones endógenas, generadas, por tanto, desde dentro, con carácter autónomo y orientación integral.

\section{Replicabilidad o transferibilidad}

Un proyecto, un programa o una acción es replicable cuando sirve de referencia para desarrollar políticas, iniciativas y actuaciones similares en otros lugares. Se entiende por transferibilidad, la capacidad de una experiencia para permitir la repetición de sus elementos esenciales en un contexto distinto al de su creación, con elevadas probabilidades de éxito. En este sentido, el criterio de transferibilidad, evalúa la capacidad de una iniciativa para ser repetida en realidades diferentes a la originaria. Es por ello que la posibilidad de réplica o transferencia se relaciona de forma estrecha con los criterios de viabilidad y factibilidad, pues dependen en gran medida de la calidad de los ajustes y políticas diseñadas en dicha experiencia; así, a mayor compatibilidad con las características del entorno, más viable será su ejecución. De ahí que no se puede entender la transferencia como una copia exacta, sino como una contextualización de dicha iniciativa en un marco de referencia diferente, por eso debe primar la simplicidad y universalidad de las ideas básicas de la iniciativa; la buena planificación de los recursos necesarios y el uso eficiente de los recursos.

La factibilidad, recientemente aludida en relación a la transferibilidad de una práctica, responde a las posibilidades de éxito que una actividad tendrá en su implementación, puesto que se ha tenido en cuenta el contexto económico, técnico, organizacional y socio-político en el diseño de dicha actuación. El criterio de factibilidad evalúa la adaptación de la iniciativa desarrollada a las necesidades y a los recursos del contexto.

A la hora de tener en cuenta la viabilidad de una experiencia, se han de considerar las siguientes dimensiones:

- Viabilidad económica: eficacia y economía de los recursos disponibles.

- Viabilidad interna: consenso en el equipo responsable de la iniciativa y aceptación entre los miembros de la organización.

- Viabilidad técnica: adecuación de las propuestas efectuadas a los medios técnicos disponibles.

- Viabilidad medioambiental: sostenibilidad, preservación de los recursos medioambientales.

- Viabilidad social: adecuación a las necesidades derivadas del contexto físico y del contexto temporal, así como a las necesidades y demandas expresadas por la ciudadanía. 
A estos cuatro criterios básicos determinados por la UNESCO (2003) para identificar "buenas prácticas", se pueden añadir otros complementarios que ayudan a perfilar mejor el tipo de experiencias que se buscan en función del ámbito al que se circunscriban. En el caso de la acción comunitaria, resulta imprescindible incorporar como indicadores del buen hacer, entre otros, la transversalidad y la implicación de la ciudadanía, pues son señas inequívocas de su identidad (Úcar y Llena, 2006).

\section{Transversalidad-integralidad}

Refiere un estilo de trabajo globalizador, interdisciplinar e interprofesional, que integra diferentes áreas de conocimiento y temáticas; permitiendo interrelacionar distintos saberes con el fin de favorecer una acción comunitaria lo más integral posible y tener una visión de conjunto. Se trata, en definitiva, de intervenir de forma holística sobre el conjunto de factores estructurales que configuran la realidad social en/con la que se está trabajando. Este criterio obligará a revisar las metodologías empleadas, puesto que se abordarán cuestiones desde diferentes ópticas disciplinares y por diferentes profesionales con el fin de dar respuestas complejas y entrelazadas.

\section{Implicación de la ciudadanía-empoderamiento-trabajo en red}

Las buenas prácticas en acción comunitaria no suelen ser individuales, sino que dependen de la implicación de varios agentes y estructuras, por lo que encuentran en los procesos participativos un elemento clave. Suponen la colaboración entre profesionales, entidades y ciudadanía; un trabajo en red capaz de crear ilusión, movilización, adhesión y compromiso generador de pequeños cambios que mejoran la calidad de vida y el bienestar individual y comunitario (FEAPS, 2007). La diversidad de agentes implicados supone un mayor esfuerzo en las tareas de coordinación, pero aporta una visión más amplia de la realidad, profundidad de la participación y conocimiento de la práctica, aportándole calidad.

Para entender que una Buena Práctica cumple este criterio, hemos de tener en cuenta tres niveles diferentes de participación ciudadana:

- Presencia: es el nivel de participación menos intenso y más marginal. Se trata de comportamientos esencialmente receptivos o pasivos, como la asistencia a reuniones o la exposición voluntaria a los mensajes políticos.

- Activación: estadio que supone un mayor compromiso, en la medida en que cada persona desarrolla una serie de actividades positivas de las cuales es delegado permanente, se encarga o es promotor.

- Participación: supone la asunción de una auténtica corresponsabilidad en las iniciativas, pues el individuo contribuye de forma directa o indirecta en la toma de decisión y en el desarrollo de la acción.

La participación que se busca es, pues, aquella relacionada con el empoderamiento, con procesos de reafirmación de la autonomía y la confianza individual y colectiva que deriva no sólo en una capacidad de decisión responsable, sino también en una incorporación plena en la sociedad de la que se forma parte. Esta corres- 
ponsabilidad ciudadana como principio básico de la acción comunitaria tiene una dimensión ética incuestionable, pues las iniciativas deben de ser respetuosas con los intereses y deseos de los participantes, coherentes en todo momento con los principios de conducta social y profesional en consonancia con el marco normativo vigente (OIT, 2003). El código ético de las instituciones, entidades y colectivos implicados es el orientador de las metodologías y de la implementación de la práctica (Agencia Andaluza de Evaluación Educativa, 2012).

\section{Identidad-cohesión social-transformación}

Las iniciativas que se promuevan han de ir orientadas al fomento de un compromiso cívico que ayude a superar las visiones individualistas para que todas las personas se sientan integrantes de la comunidad; así, partiendo de las particularidades y singularidades de los sujetos, se impulsará la construcción de una identidad común en aras de lograr un entendimiento mutuo que ayude a entretejer la cohesión social y el máximo bienestar.

Esto implica que las diferentes intervenciones socioeducativas -sustentadas en la identidad colectiva- han de cristalizar en procesos que contribuyan al progreso y cambio social; articulando estrategias que favorezcan la participación de los distintos agentes y movimientos sociales con el fin de responder a las necesidades y demandas comunitarias.

\section{Evaluación comunitaria}

En toda práctica social debería de incorporarse la dimensión evaluativa para seguir investigando y mejorando la calidad de los procesos que se llevan a cabo con los grupos y las comunidades. Así, a través de esta praxis se generan nuevos conocimientos de la realidad en la que se desarrolla una determinada intervención, posibilitando cambios orientados a la participación y evaluación de las acciones comunitarias; que -en palabras de Núñez y otros (2014)- atenderán a la dimensión colaborativa, participativa-práctica, participativa-transformadora y para el empoderamiento.

Esta dimensión evaluativa debería de contar con la colaboración de diferentes agentes y actores sociales, desde las administraciones públicas y organizaciones civiles, hasta la propia ciudadanía; al posibilitar su incorporación en el diseño, implementación y evaluación de iniciativas socioeducativas. Como diría Mertens (2011), son estrategias de evaluación-intervención que han de asumir la reciprocidad en las actuaciones entre evaluadores y las personas de las comunidades; la validación de los conocimientos y la experiencia de las comunidades; la sostenibilidad de la práctica evaluadora y el reconocimiento de los límites de la evaluación.

\section{Los Bancos de buenas PRÁCticas. Una oportunidad PARA ENCONTRAR REFERENTES}

A este respecto, con el objetivo de facilitar el intercambio de experiencias innovadoras y creativas para el desarrollo de los contextos y las comunidades, se crean 
unas bases de datos que -a modo de Banco de Buenas Prácticas (BBP)- difunden iniciativas que repercuten en la mejora de la calidad de vida de los entornos y las personas. Además son plataformas de divulgación de una gran diversidad de experiencias representativas de la acción socioeducativa de un territorio, y posibilitan la sistematización y promoción de acciones, programas o proyectos que -directa o indirectamente- contribuyen al desarrollo local de las comunidades, poniendo especial énfasis en aquellas dimensiones, ámbitos y/o colectivos que requieren de una atención más específica en aras de mejorarlas.

Los BBP representan una importante fuente documental, una guía y un apoyo para el trabajo de aquellos profesionales que desarrollan su labor investigando, diseñando, planificando, gestionando y ejecutando actuaciones novedosas en administraciones, entidades, organizaciones, etc. Se basan en las tecnologías de la información y la comunicación para publicitar, dar a conocer, fomentar el intercambio de experiencias así como incentivar el trabajo en red entre los agentes que pertenecen a comunidades y/o entidades -de carácter público o privado- que presentan características similares.

En este espacio de intercambio tendrán cabida aquellas experiencias innovadoras y exitosas que estén orientadas a mejorar la realidad social y que contribuyan al fortalecimiento de una actuación integral e integradora en el conjunto de la comunidad.

\section{Qué pretenden}

En general, los Bancos de Buenas Prácticas tienen como objetivos los siguientes:

- Compilar y sistematizar información sobre iniciativas y actuaciones desarrolladas por diferentes entidades, con el fin de que puedan ser transferidas a otras comunidades adaptándolas a las características y particularidades de cada territorio y/o escenario social.

- Servir de fuente documental para políticos, técnicos, investigadores y formadores en los diferentes ámbitos de lo social.

- Evidenciar la pluralidad de iniciativas y/o actuaciones atendiendo a la diversidad de entidades, temáticas, colectivos y territorios.

- Profundizar en el diseño, gestión, planificación y desarrollo de contribuciones especialmente significativas y relevantes.

- Impulsar canales de comunicación que posibiliten la divulgación de experiencias vanguardistas que representan indicadores de futuro para otros contextos y/o realidades.

- Facilitar el intercambio de información, la cooperación local y el trabajo en red para el diseño de políticas municipales.

- Conectar con otros Bancos de Buenas Prácticas.

\section{Proceso y sistematización}

Las buenas prácticas se identifican con los actuales planteamientos en torno a los criterios de calidad y eficiencia de las intervenciones sociales; y no sólo atienden a la gestión y los procedimientos, sino también a la satisfacción de las necesidades de las 
personas y las comunidades así como al desafío de mejorar sus realidades cotidianas. Para ello, compilan diferentes experiencias que -basándose en múltiples perspectivas y modalidades de intervención- abarcan desde la prevención y la sensibilización hasta la coordinación y evaluación de diferentes iniciativas.

Así, tal y como exponíamos en relación a los criterios de identificación, se seleccionan experiencias que han sido implementadas en diferentes contextos y que -precisamente por su relevancia, por su originalidad, innovación, etc.- en muchos casos han merecido algún tipo de distinción. De este modo, a través de los bancos se pretende darlas a conocer e impulsar una mayor divulgación, con el fin de que puedan ser adaptadas y transferidas.

En lo fundamental, para localizar ejemplos de buenas prácticas hemos de recurrir a bancos de documentos, cuadernos y concursos de experiencias, observatorios vinculados a alguna institución y/o entidad pública o privada, a las programaciones municipales que -si bien realizan muchas iniciativas- a veces no trascienden más allá del contexto social más cercano. Pero también podemos aproximarnos a ellas a través de los medios de comunicación (prensa, radio y televisión), ya que en ocasiones se informa de actuaciones puntuales que -por su carácter innovador y pioneromerecen un lugar destacado en las noticias diarias.

Con todo, resulta bastante frecuente encontrarnos con barreras que dificultan la detección e identificación de prácticas que son consideradas como 'buenas' o 'excelentes'. Muchas de estas limitaciones residen en el desconocimiento, en la escasa información aportada, en la falta de sistematización y concreción, en la desconfianza respecto de los informantes y/o promotores de las iniciativas o, simplemente, debido a la escasa consideración que se otorga a este tipo de transferencias.

En definitiva, para identificar y seleccionar aquellas experiencias prácticas implementadas y que más se adecuan a unos estándares de innovación, eficiencia, sostenibilidad y replicabilidad (UNESCO, 2003), han de establecerse ciertos criterios que permitan homologar su presentación; por ello, el hecho de utilizar un vocabulario común y coherente para todos será fundamental a la hora de explicar la iniciativa y compartir los procesos puestos en marcha y que se convierten en el germen de un cambio positivo en los métodos de actuación tradicionales. Además, la información debería adoptar formatos adecuados, comparables y transferibles con el fin de facilitar su utilización.

La presentación de las buenas prácticas responde, por lo general, a un esquema semejante al que a continuación se expone:

- Datos de la Experiencia: en el que se indicará el título de la misma, que deberá ser conciso pero significativo respecto al contenido abordado en la Buena Práctica; el ámbito temático al que se adscribe la actuación y las palabras clave que la identifican. Además, debería indicarse quién es el agente promotor así como la entidad y naturaleza jurídica; también se explicitan los datos de localización y de la persona de contacto.

- Descripción y características de la buena práctica: en este apartado, se hará una breve introducción de la experiencia, donde -en pocas líneas- se describirá lo esencial con el fin de facilitar una primera y rápida aproximación a su contenido, así como la explicación de los antecedentes que motivan esta 
actuación. Al tiempo, se enumeran los objetivos, que han de ser reales, palpables, cuantificables y evaluables; los destinatarios, que han de formar parte del diseño, participando de modo directo en las diferentes fases de intervención; y el ámbito de actuación (local, regional, estatal). Además, se relacionan los agentes sociales (administraciones públicas, sindicatos, empresas, fundaciones, ONG's), la pertenencia a redes y la coordinación entre diferentes entidades que contribuyen a la implementación de dicha práctica.

En este apartado también se explicitará la metodología necesaria para poder desarrollar dicha experiencia y que, en lo fundamental, ha de ser creadora, aglutinadora, práctica y participativa. Una metodología que necesariamente ha de trascender al diseño, pues es pertinente contemplar el proceso de evaluación y difusión de la iniciativa; valorando especialmente elementos innovadores como la intersectorialidad, la multidisciplinariedad, la coordinación, etc. En definitiva, explica el modo mediante el cual -partiendo del problema- se avanza hacia la consecución de los objetivos; esto es, el recorrido del cambio y sus claves racionales.

- Recursos para la implementación: en este bloque de información, se indicarán los recursos humanos, económico-financieros así como los medios infraestructurales necesarios para poder implementar dicha iniciativa.

- Resultados y beneficios: aquellos que están directamente relacionados con la consecución o no de los objetivos previstos, y que han de ser cuantificables, desarrollando instrumentos y mecanismos que impulsen la difusión de resultados. Se describirán los efectos finales de las actuaciones en términos de mejoras objetivas.

- Dificultades en la implementación: en él se indicarán los problemas técnicos, financieros, infraestructurales... que se han producido; así como las medidas adoptadas para minimizar los obstáculos surgidos durante su desarrollo.

- Evaluación: se explicitan las técnicas utilizadas para analizar en qué grado se responde a los objetivos planteados, a las necesidades que presentan las problemáticas estudiadas así como los indicadores asociados a dichos logros; esto es, la influencia en el territorio, la implicación de diferentes agentes locales, la interterritorialidad, etc.

- Transferencia: se resumirá lo esencial de la buena práctica y las lecciones derivadas de la experiencia, de tal modo que pueda ser de utilidad para otros.

- Documentación: se adjunta toda aquella documentación (fotografías, folletos, póster, noticias de prensa...) que permite visualizar el desarrollo e implementación de la iniciativa.

A continuación, presentamos dos bancos que compilan experiencias poseedoras de los requisitos para ser consideradas como una "buena práctica"; entendidas no sólo como prácticas "buenas" sino como iniciativas que -desde una perspectiva integral e integradora- impulsan actuaciones que promueven y dinamizan diferentes ámbitos de la Pedagogía-Educación Social mediante metodologías innovadoras que favorecen la participación, la cohesión y la transformación social. 


\section{A MODO DE EJEMPLO}

\section{Banco Internacional de Documentos de Ciudades Educadoras}

http://www.bcn.cat/edcities/aice/estatiques/espanyol/sec_banc.html

El movimiento de Ciudades Educadoras se inició en Barcelona en el año 1990 con el objetivo de trabajar conjuntamente en proyectos y actividades municipales que permitiesen mejorar la calidad de vida de los habitantes, partiendo de una implicación activa en el uso y la evolución de la propia ciudad, de acuerdo con la carta aprobada de Ciudades Educadoras.

El Banco Internacional de Documentos de Ciudades Educadoras - gestionado por la AICE- contiene dos bases de datos complementarias: una de búsqueda de experiencias prácticas que tratan diferentes temáticas y que están orientadas a ciudadanos de todas las edades; y otra de documentos y lecturas recomendadas que sirven de apoyo para el desarrollo del concepto de Ciudad Educadora. Asimismo, en esta página se destacan temas de actualidad y prácticas innovadoras que sirven de referente para poner en marcha actuaciones específicas en ciudades similares.

En cada una de estas bases de datos, tenemos la opción de seleccionar el área geográfica (continente, país, ciudad o localidad) en la que queremos centrar nuestra búsqueda, así como los descriptores generales y específicos que pretendemos resaltar para cada uno de estos ámbitos. Entre los generales encontramos, entre otros, los siguientes: arte y humanidades, bienestar social, civismo y convivencia, desarrollo socioeconómico, política y administración, sistema educativo, asociacionismo y participación, cultura y ocio, salud y deportes.

Cada experiencia se presenta con una ficha que contiene la información necesaria para comprender el porqué de su implementación; esto es, la ciudad donde se lleva a cabo, el número de habitantes, el país y continente donde se localiza, los temas que directa o indirectamente se trabajan a través de la puesta en práctica de esta experiencia así como los grupos de edad a quienes va dirigida. Además, sitúa el período en el que se ha desarrollado (año de inicio y de finalización) así como los principios de la carta internacional de ciudades educadoras a los que se refiere.

A continuación, se presenta una breve justificación y resumen de la experiencia que da cuenta de los objetivos, metodología, contexto y estrategia de evaluación que se ha seguido en su puesta en marcha; así como otra información adicional (fotografías, vídeos, carteles) que ayudan a ejemplificar dicha práctica, facilitando los datos de contacto de los responsables de su ejecución.

\section{Programa Buenas Prácticas HABITAT II}

http://habitat.aq.upm.es/lbbpp.html

El Programa de Buenas Prácticas nace en junio de 1966 en Estambul (Turquía) en el seno de la Segunda Conferencia de Naciones Unidas sobre Asentamientos Humanos (HABITAT II). Así, los Comités Nacionales de diversos países se propusieron reunir ejemplos de prácticas que respondiesen a los objetivos de la Conferen- 
cia, como una forma de identificar políticas y actuaciones urbanas que se hubiesen mostrado eficaces - desde criterios de sostenibilidad- para mejorar las condiciones de vida en las ciudades y pueblos.

En este banco nos encontramos dos bloques: uno vinculado a catálogos de experiencias y otro de documentos relacionados con la Conferencia y boletines informativos. De este modo, se reúnen diferentes compilaciones de iniciativas españolas, latinoamericanas, concursos internacionales y un buscador específico, que posibilita delimitar el año, el tipo de práctica (award, best, good), la región geográfica (América, Asia, Oceanía, Europa, África y Estados Árabes), el tipo de institución que la promueve (administraciones, ONG, asociaciones, sector privado, etc.), la categoría (atendiendo a 17 descriptores, tanto temáticos como de colectivos), la escala de actuación (a diferentes niveles, desde un alcance mundial a otro más específico como puede ser un barrio), así como el ecosistema en el que deseamos centrar nuestra búsqueda.

Éstos tan sólo son dos ejemplos entre los muchos bancos de buenas prácticas existentes; en general, presentan esta doble estructura de sistematización de experiencias y catálogos de documentos que impulsan el conocimiento de actuaciones innovadoras, potencian la difusión y transferencia de buenas prácticas locales y favorecen el trabajo en red, alentando la reflexión sobre la práctica.

\section{Consideraciones finAles}

Circunscribiéndonos al ámbito de la acción comunitaria, caracterizado por un fuerte componente interdisciplinar, una revisión de lo que son las buenas prácticas -acompañada de una búsqueda de las iniciativas catalogadas como tales y recogidas en bancos gestionados por diferentes instituciones- es suficiente para constatar que no se requieren actuaciones muy ampulosas ni infinidad de medios, sino más bien una cierta dosis de sentido común y de capacidad para rentabilizar al máximo los recursos disponibles capaces de generar una respuesta integral a las necesidades sociocomunitarias; poner en valor el trabajo cotidiano desde una cultura de la reflexión y de la sistematización para crear conocimiento válido, contrastado, documentado, transferible y útil.

Se trata, en definitiva, de experiencias dotadas de originalidad que inspiran el concepto de una buena práctica transferible, sustentable e innovadora, homologable a determinados territorios. De ahí su potencialidad para poner en evidencia nuevas formas de incrementar la creatividad, reinventando determinadas actuaciones socioeducativas en aras de conseguir una cultura más pedagógica, más cívica y más ciudadana.

Afirma Marco Marchioni (2014: 10) -reconocido trabajador e investigador socialque la crisis económica y sus consecuencias "han puesto en evidencia las carencias y los errores de las políticas sociales que se han desarrollado a lo largo de demasiado tiempo en nuestro país". Entre ellas identifica una visión fragmentada de la acción social, con unas actuaciones fundamentalmente centradas en las demandas individuales, ignorando que "las consecuencias sociales son individuales, pero las causas son colectivas" (ibid.); unas políticas sociales orientadas hacia las personas, pero que, en su mayoría, se han desarrollado sin contar con ellas; por último, una deficiente 
coordinación entre administraciones y de éstas con el tercer sector. Todo ello se resume en una ausencia de visión comunitaria en las políticas sociales, obviando en el trabajo cotidiano los indicadores que, a nuestro entender, debe satisfacer cualquier iniciativa que pretenda ser considerada como buena práctica en este ámbito: la transversalidad e integralidad de las acciones, la implicación de una ciudadanía empoderada -también en los procesos evaluativos de carácter comunitario- y el trabajo en red. Todos ellos redundarán en la construcción de comunidades más cohesionadas y desarrolladas en clave humana.

\section{REFERENCIAS BIBLIOGRÁFICAS}

Agencia andaluza de evaluación educativa (2012). Guía de Buenas Prácticas Docentes. Junta de Andalucía: Sevilla. Recuperado de: http://goo.gl/LNyvTW.

Confederación española de organizaciones a favor de las personas con discapacidad intelectual (FEAPS) (2007). Buenas prácticas FEAPS ¿Qué son y cómo se valoran? Recuperado de: http://goo.gl/fmL1Za.

Fundación CEPAIM. Convivencia y cohesión social (2014). Herramientas para la cohesión social. Un proceso de reflexión y sistematización entre buenas prácticas en acción comunitaria intercultural de España y Portugal. Murcia: Fundación CEPAIM.

Marchioni, M. (2014). Políticas sociales y dimensión comunitaria. Revista voces inmigrantes, 20, 10-11.

Mertens, D.M. (2011). Social transformation and evaluation. Evaluation Journal of Australasia, 10(2), 3-10.

Núñez, H., Crespo, E., Úcar, X., y Llena, A. (2014): Enfoques de evaluación orientados a la participación en los procesos de acción comunitaria. Pedagogía social. Revista interuniversitaria, 24, 79-103.

Observatorio internacional de la democracia participativa (2015). IX edición de la distinción OIDP "Buena práctica en participación ciudadana impulsada desde los gobiernos locales". Recuperado de: http://goo.gl/CqMJfF.

Organización Internacional del Trabajo (2003). Directrices sobre buenas prácticas: identificación, análisis, estructuración, difusión y aplicación. Programa internacional para la erradicación del trabajo infantil. Recuperado de: http://goo.gl/Q1Rmf8.

Úcar, X., y Llena, A. (2006, coords.). Miradas y diálogos en torno a la acción comunitaria. Barcelona: Graó.

UNESCO (2003). Best practices. Recuperado de: http://www.unesco.org/most/bphome. htm\#1. 